\title{
Multimodal Images Integration for Catheter Ablation of Ventricular Tachycardia
}

\author{
N Courtial ${ }^{1,2}$, A Simon ${ }^{1,2}$, M Lederlin $^{1,3}$, S Bruge $^{1,2}$, RP Martins $^{1,3}$, M Garreau $^{1,2}$ \\ ${ }^{1}$ Université de Rennes 1, LTSI, Campus de Beaulieu, Rennes, F-35042 \\ ${ }^{2}$ INSERM, U1099, Campus de Beaulieu, Rennes, F-35042 \\ ${ }^{3}$ CHU Rennes Pontchaillou, Rennes, F-35700
}

\begin{abstract}
Radiofrequency catheter ablation is an important therapeutic option for patients with recurrent ventricular tachycardia $(V T)$. The procedure aims at cauterizing regions of the ventricle. A good knowledge of anatomical structures and tissues properties can improve procedure safety and efficacy. This work aimed at analysing the feasibility of a workflow based on multimodal image integration to assist the intervention. We focused here on a model including myocardial thickness and fibrosis extracted from late gadolinium enhanced MRI (LGE-MRI) sequence, and left ventricular anatomy segmented from multiphase computed tomography $(C T)$. The produced model can be imported within Carto-3 ${ }^{\circledR}$ system (Biosense Webster Inc, Diamond $B a r, C A)$, the software used during the ablation procedure. Four patients cases have been included in this preliminary study. The four produced patient specific models have been validated by a clinician, and two of them have been integrated within Carto- $3^{\circledR}$ system and used during the intervention.
\end{abstract}

\section{Introduction}

Catheter ablation, used for 30 years, aims at cauterizing ventricle's regions responsible for VT initiation and maintenance. To do so, a localized delivery of radiofrequency energy is applied. It's an important therapeutic option for patients suffering of recurrent VT resistant to antiarrythmic drugs. Depending on the needs, an endocardial or an epicardial approach can be considered. Ablation of ventricle regions is not an innocuous procedure, and it raises important questions to proceed safely and efficiently. During the procedure, the physician has to know (i) the catheter's position against the VT sources, (ii) which spots to avoid (arteries, phrenic nerve), (iii) which regions could be potential targets, and (iv) what constraint can the catheter apply on the cardiac wall without perforating it (myocardium thickness).
Answers have been found to these different requirements. Electroanotomical mapping systems have been early recognized as a powerful assistance tool for VT ablation [1]. Studies such as [2] have shown the benefit of visualizing structures at risk during VT ablation, segmented from CT series. A multimodal model integration has been proposed in [3], including both CT and MRI enhanced sequences. It contains wall thining (WT) zones, defined as zones where myocardium wall's thickness is less than 5 $\mathrm{mm}$ [4] segmented from CT, and scare core and grey zone, defined as more than $50 \%$ and $35-50 \%$ of maximal myocardial signal [5] respectively. The interest of such informations has been later confirmed in [6], as a good predictor of local abnormal ventricular activity (LAVA).

Multimodality has been recognized in different heart surgery as a powerful tool to plan and guide interventions [7][8][9]. We propose here a patient specific multimodal model built from CT and LGE-MRI, images including the left endocardium segmented on CT, and fibrosis and myocardium's thickness segmented on LGE-MRI. LGE-MRI is registered on CT in a process including Cine-MRI as an intermediate step. The objective is to use CT informations as anatomical landmarks, fibrosis locations as LAVA predictors, and myocardium thickness as a safety information, to prevent catheter from perforating myocardium's wall.

In this paper, we present a workflow allowing to create patient specific model compatible with the used procedure during TV's ablation, regarding the process time, and the used technology. The descriptors are all segmented in semi-automatic processes. The fusion of these descriptors and the model creation are fully automatic. Both methods and data visualizations have been merged within a single software solution, making this workflow user friendly, and a time-saving system.

\section{Methods}

For each patient, CT, LGE-MRI, and Cine-MRI have been considered. The workflow is presented in figure 1 . 


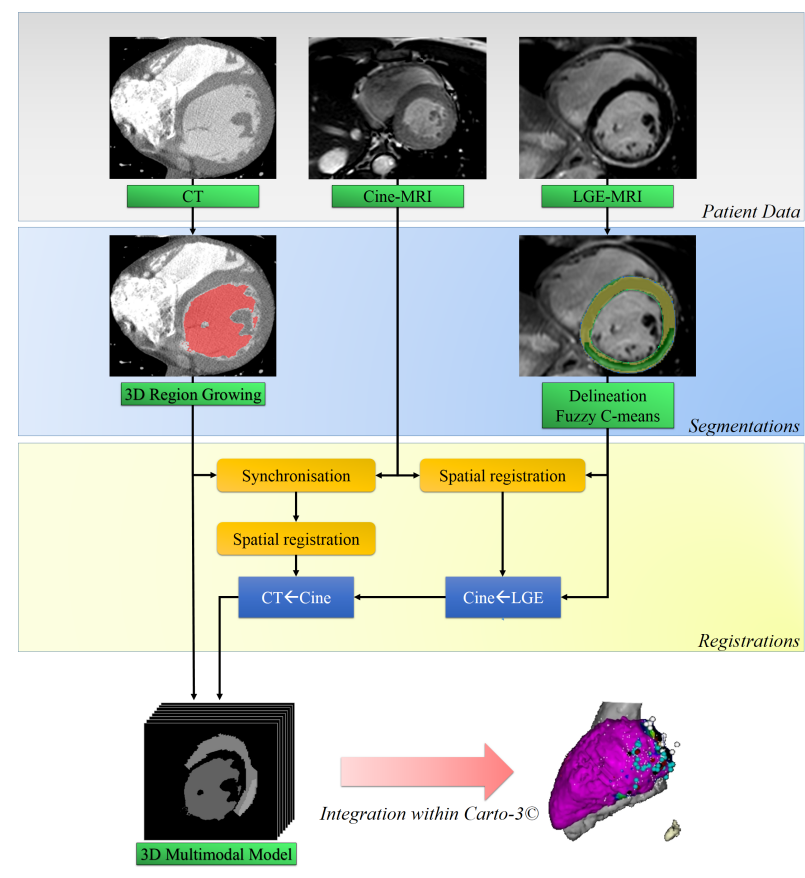

Figure 1. Complete workflow, from images acquisition, to model integration

\subsection{Segmentation}

The segmentations on the CT images and LGE-MRI sequence work independently in a semi automatic process.

On the CT images, the left ventricle's cavity is well constrasted, since a contrast agent has been used. Thus, it's been segmented by using a 3D region growing, followed by a morphological closing. The parameters have been optimised to provide good results on the images obtained at CHU Rennes Pontchaillou (GE Healthcare Discovery CT750 HD), so the only required interaction is the choice of the seed(s).

On the LGE-MRI, a manual delineation of the endocardial and the epicardial contours is performed on each available slice in short axis view (SAX). The user picks control points on which a spline is computed. An average of 6 and 10 points are needed to define endocardial and epicardial contour respectively. Then, the healthy parts and the fibrosis are segregated using a fuzzy C-means algorithm [10] within the defined myocardium annulus.

\subsection{Registration}

The extracted informations are represented in the CT's referential, as this is the modality with the highest spatial definition.

Cine-MRI is used in order to improve the registration robustness, firstly by relying on both Cine-MRI high tem- poral resolution and its closeness to LGE-MRI, and then by performing a multi-images registration with $\mathrm{CT}$ sequence.

Cine-MRI and CT sequences are registered in a multiimages process, i.e. a single transformation is optimized to register multiple couples of images. The two sequences are imaging the heart along the RR-interval, at evenly distributed instants. However, these two sequences being taken during different medical exams, RR-interval variations occur, resulting in temporal distortions, e.g. the diastolic duration in regards of the RR-interval [11]. As a consequence, a non linear synchronisation has to be performed. Here a Dynamic Time Warping (DTW) has been chosen.

ECGs being unavailable, an image-based descriptor is used to perform the DTW. Normalized cross correlation (NCC) has been used as a descriptor to describe the cardiac dynamics along the sequence. A curve representing the NCC between the first image, corresponding to the peak of the wave R, and the rest of the series along a cardiac cycle is computed separately for both sequences. The DTW is then performed on the obtained curves.

The used algorithm, described in [12], consists into finding the time warping function putting the two NCC curves as close as possible, by associating all the indexes of the poorest defined temporally sequence (CT), to indexes of the second one. To ensure realistic results, the available domain has been limited, and the step pattern has been adapted in regards of the temporal resolution ratio of the two series. A Djikstra algorithm has been used to find the minimum path. A result of this synchronisation can be seen in figure 2.

A rigid transform is then computed using a NMI based multi-images registration between the full CT sequence, and the Cine-MRI volumes taken at the corresponding phases.

Despite LGE-MRI and Cine-MRI sequences are acquired during the same medical exam, they are commonly misaligned. In order to compensate that, a rigid registration is performed between the two sequences. LGE-MRI being a non temporal series, a single phase is taken from Cine-MRI, the closest from the LGE-MRI's one, considering the temporal distortion within the cardiac cycle to be negligeable between the two sequences as their acquisition conditions are particularly close regarding patient's state [13]. The registration is computed using the normalized mutual information (NMI) as similarity measure [14].

\subsection{Patient specific model production}

The patient specific model is then created from the CT and the LGE-MRI labelled images. First, the LGE-MRI labelled image is resampled using the combination of the LGE/Cine-MRI registration and the Cine-MRI/CT one. Once in the same geometrical referential, both images are 


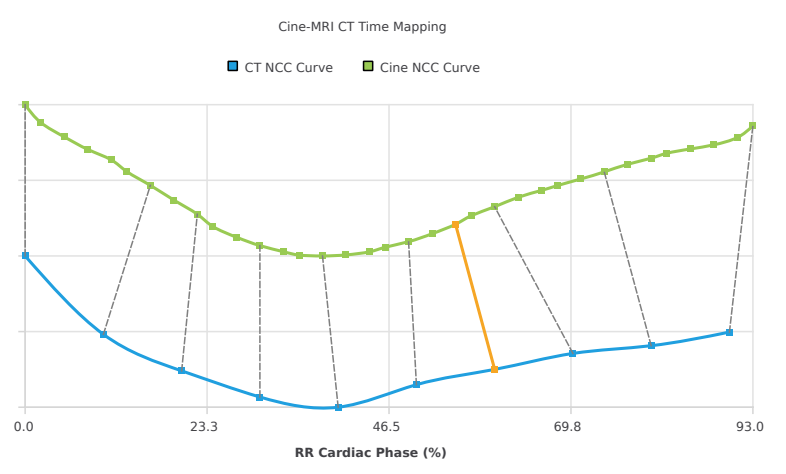

Figure 2. Result of DTW

fused by keeping the voxel of highest value. The label values have been chosen to be higher following their considered criticality, here: Endocardium, Myocardium, Fibrosis in ascending order. Once the synthetic DICOM series produced, the headers from the original CT are copied to be recognized as a genuine medical exam. For safety reason, UIDs are regenerated, and the exam name is modified in order to make obvious this is a synthetic series once imported in the electrophysiology lab.

\section{Results}

This feasability study has been conducted on a 4 patients dataset. Considering the work done on MRI images, patients with defibrillators and no available MRI sequences have been excluded.

Both segmentations and registrations were visually validated by a clinician. If checkerboard is the classical way of evaluating registrations, a 3D visualization of endocardial cavity and the LGE SAX view slices has been used in addition (figure 3). The segmentations, the registrations, the visual checks, and the synthetic DICOM series generation have all been included in a single software solution, in order to make the whole process easier and quicker. If a registration had to be wrong, it can still be modified manually. On the four test cases, none have required such a process, despite a particularly poor MRI quality for one of them.

The images processing has a total duration of about 15 minutes per patient, including:

- Left ventricle cavity segmentation (CT): 2 minutes

- Endocardium and Epicardium delineation (LGE-MRI): 2 minutes

- Automated registrations: 5 minutes

- Registration checks: 5 minutes

This processing time makes this workflow perfectly compatible with the clinical schedule.

During the procedure, Carto- $3^{\circledR}$ system is used. It allows to embed segmentations obtained from CT images.
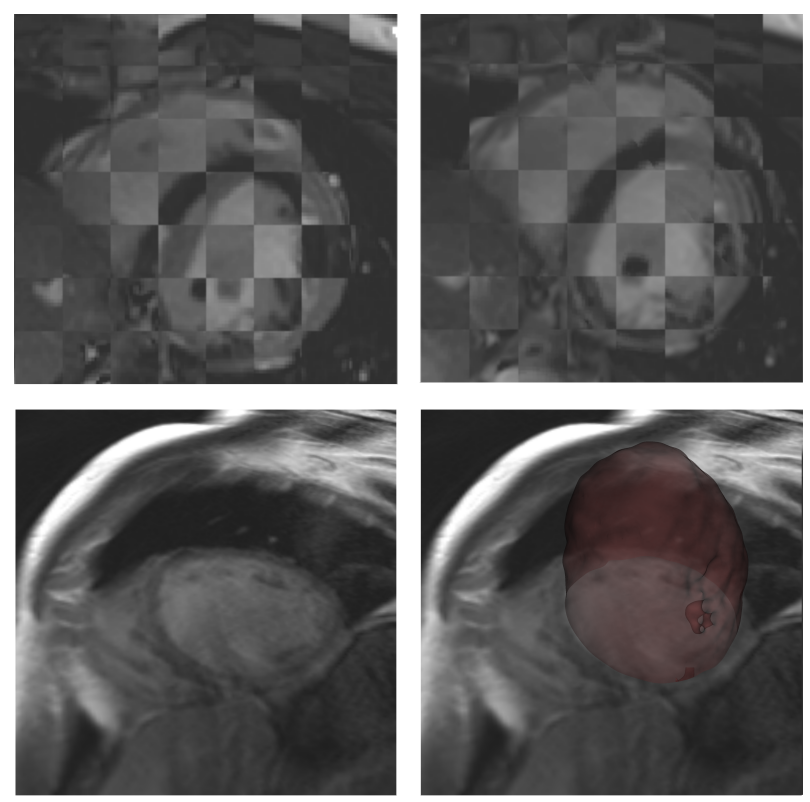

Figure 3. Top: Checkerboard of LGE-SAX (darker) and Cine-SAX: Left: Before Registration; Right: After Registration; Bottom: LGE-SAX slice and 3D model of left ventricle's cavity in transparency

Here, the fused descriptors have been exported into a synthetic DICOM series, extending the system capability to multimodal model. The obtained 3D model is manually registered with the electroanatomical mapping during the procedure.

The produced model has been integrated within Carto$3^{\circledR}$ system and used during intervention for two patients. The obtained model (split in two parts for readability purpose) is shown in figure 4.

\section{Conclusion}

This work shows that bringing synthetic multimodal models to the electrophysiology lab during catheter ablation of VT is feasible without changing the existing procedure. The presented workflow could be speed up by reducing the number of needed interaction. Particularly, the delineation work on LGE-MRI SAX view could be automated such as seen in [15]. During the intervention, thickness information confirmed to be a driver of clinician's confidence when ablating, as the safety margin before perforating cardiac's wall was known.

Our future aim is to include more patients to evaluate the contribution to procedural efficacy. We'll consider extracting myocardium thickness from the CT images. This would allow to consider more patients, making the ones with defibrillators, used as a safety net for people suffering from VT, compatible with the presented workflow, and to 

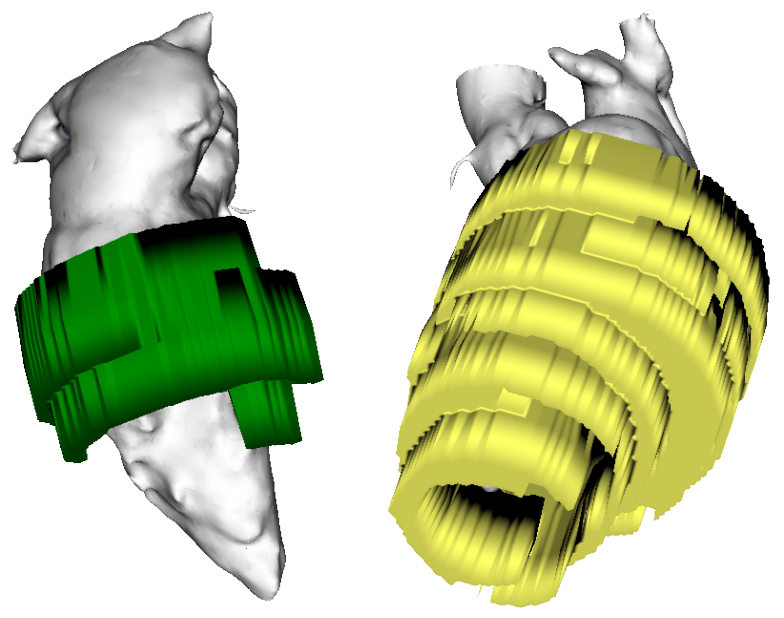

Figure 4. Left: Left ventricular endocardium (CT) and fibrosis areas (LGE-MRI); Right: Left ventricular endocardium (CT) and myocardium thickness (LGE-MRI)

increase spatial definition of the thickness information. We will also consider more complex models, including structures at risks (phrenic nerve, coronary arteries).

\section{References}

[1] Stevenson, et al. Identification and Ablation of Macroreentrant Ventricular Tachycardia with the CARTO Electroanatomical Mapping System. PACE 1998;21:14481456.

[2] Yamashita, et al. The Role of High-Resolution Image Integration to Visualize Left Phrenic Nerve and Coronary Arteries during Epicardal Ventricular Tachycardia Ablation. Circulation Arrhythmia Electrophysiology 2015;8:371-380.

[3] Cochet, et al. Integration of Merged Delayed-Enhanced Magnetic Resonance Imaging and Multidetector Computed Tomography for the Guidance of Ventricular Tachycardia Ablation: A Pilot Study. Journal of Cardiovascular Electrophysiology 2013;24:419-426.

[4] Stolzmann, et al. Reference values for quantitative left ventricular and left atrial measurements in cardiac computed tomography. European Radiology 2008;18:1625-1634.

[5] Roes, et al. Infarct tissue heterogeneity assessed with contrast-enhanced MRI predicts spontaneous ventricular arrhythmia in patients with ischemic cardiomyopathy and implantable cardioverter-defibrillator. Circulation Cardiovascular Imaging 2009;2:183-190.

[6] Yamashita, et al. Image Integration to Guide Catheter Ablation in Scar-Related Ventricular Tachycardia. Journal of Cardiovascular Electrophysiology 2016;27:699-708.

[7] Roes, et al. Imaging and atrial fibrillation: The role of multimodality imaging in patient evaluation and management of atrial fibrillation. European Heart Journal 2010;2:542551.

[8] Mahara, et al. Multimodal assessment of coronary obstruc- tion for transcatheter aortic valve implantation. European Heart Journal Cardiovascular Imaging 2015;16:1171.

[9] Tavard, et al. Multimodal registration and data fusion for cardiac resynchronization therapy optimization. IEEE Transactions on Medical Imaging 2014;33:1363-1372.

[10] Kachenoura, et al. Robust assessment of the transmural extent of myocardial infarction in late gadolinium-enhanced MRI studies using appropriate angular and circumferential subdivision of the myocardium. European Radiology 2008; 18:2140-2147.

[11] Chung, et al. Duration of diastole and its phases as a function of heart rate during supine bicycle exercise. American journal of physiology Heart and circulatory physiology 2004;287:H2003-H2008.

[12] Betancur, et al. Synchronization and Registration of Cine Magnetic Resonance and Dynamic Computed Tomography Images of the Heart. IEEE Journal of Biomedical and Health Informatics 2016;20:1369-1376.

[13] Shekhar, et al. Registration of real-time 3D ultrasound images of the heart for novel 3D stress echocardiography. IEEE Transactions on Medical Imaging 2004;23:11411149.

[14] Betancur, et al. Registration of dynamic multiview 2D ultrasound and late gadolinium enhanced images of the heart: application to hypertrophic cardiomyopathy characterization. Medical Image Analysis 2016;28:13-21.

[15] Kurzendorfer, et al. Fully automatic segmentation of left ventricular anatomy in 3D LGE-MRI. Computerized Medical Imaging and Graphics 2017;59:13-27.

Address for correspondence:

Nicolas Courtial

LTSI / Université Rennes 1

Campus de Beaulieu / F-35042 Rennes / France

tel.: +33-2-2323-6220

nicolas.courtial@univ-rennes1.fr 